\title{
Single Atom Imaging and EELS; 40 Years Ago
}

\author{
M. S. Isaacson \\ University of California at Santa Cruz, Jack Baskin School of Engineering, \\ Department of Electrical Engineering, Santa Cruz, Ca. USA msi@soe.ucsc.edu
}

New discoveries and ideas often occur at the confluence of events and technologies that allow them to happen. So it was with the development of the first electron microscopic observations of individual atoms at the University of Chicago laboratory of Albert Crewe four decades ago. The idea of the Scanning Transmission Electron Microscope was not new, having been discussed by Von Ardenne (1) in 1938. The idea of using a field emission gun in a scanning microscope was implemented by Zworykin in 1942, although in a sealed glass tube (2). Hillier and Baker demonstrated the use of energy analysis in the electron microscope in 1944 (3). And certainly, ultra high vacuum systems (UHV) had been around for decades.

It was the combination of all of these ideas that fueled the ideas of Albert Crewe, a former particle accelerator physicist. His knowledge of particle optics and only a vague familiarity with electron microscopy, led him to assemble those ideas first at Argonne National Laboratory (where he was the director). Then at the University of Chicago with a small group of students, they put together a cold field emission source coupled to an aberration optimized two electrode acceleration system (fig.1), i.e., the "Butler" gun (4), a UHV system pumped by two 4001/sec ion pumps, a single low aberration side-entry magnetic lens, a series of annular detectors and an electrostatic energy spectrometer which would allow for energy filtering and simultaneous collection of elastic, inelastic and unscattered electrons (fig.2). Simultaneously, the group also designed and constructed an SEM using the "Butler" gun as the only lens in order to test their knowledge of the optics and in which to develop an electron spectrometer partially corrected for $2^{\text {nd }}$ order abberations (5). Later, the group would be joined by Elmar Zeitler and Mike Thomson who put the optical theory of the STEM on firm footing (6).

The system shown in figure 2 operated at $30-40 \mathrm{keV}$ and in 1970 it was demonstrated that high enough contrast could be achieved to image individual thorium atoms linked by a polymer chain, using an annular dark field (ADF) detector at about $0.3 \mathrm{~nm}$ resolution (7). Using the knowledge gained from the "gun" microscope, and improving the electrical, mechanical stability and magnetic shielding of the system (8), $0.24 \mathrm{~nm}$ resolution could be attained with an ADF signal such that individual gold atoms could be easily visualized (Fig. 4) on $1 \mathrm{~nm}$ thick carbon substrates.(9) Concurrently with these developments, the group also demonstrated the potential of the EELS technique at high energy resolution (at that time, $0.25 \mathrm{eV}$ ) in being able to selectively identify chemical components of samples [Fig. 5,6 (10) ]. These initial results led to the prediction that EELS of individual atoms should be possible (11).

For years afterwards, the push in the EM community was to improve image resolution by going to higher keV and eventually to aberration correction (12). But after 40 years it had 
become apparent that because of radiation damage due to knock-on collisions (eg.13), imaging at lower $\mathrm{keV}$ would be the mode d'jour when looking at lighter element systems such as carbon nanotubes and soft materials. Performing energy loss spectroscopy on individual atoms is now a reality (e.g.,14,15), thanks to Albert Crewe's unconventional thinking 40 years ago.

References:

[1] M. von Ardenne, Z. Phys.109.553-572 (1938).

[2] V.A. Zworykin, J.Hillier and R.L.Snyder. ASTM Bull.117.15-23 (1942).

[3] J. Hillier and R.F. Baker. J. Applied Phys.13.663-675 (1944).

[4] J.W.Butler.Proc. $6^{\text {th }}$ Int. Cong. on EM, Kyoto.Vol.1.191(Maruzen. Co., Tokyo, 1966)

[5] A.V.Crewe, M.S.Isaacson and D.Johnson. Rev.Sci. Instr. 40, 241 (1969)

[6] E. Zeitler and M.G.R.Thomson. Optik.31.258-280 (1970).

[7] A.V.Crewe, J.Wall and J.P.Langmore. Science.168(3937).1338-1390 (1970).

[8] J.Wall, J.Langmore, M.Isaacson and A.V.Crewe, PNAS(USA).71(1974).1-5.

[9] M.Ohtsuki, M.S.Isaacson and A. V. Crewe,SEM.1979.II. 375-382.(SEM Inc., Ill.)

[10] M.Isaacson, J.Chem.Phys.56, 1803,1813 (1972)

[11] M.Isaacson and D.Johnson. Ultramicroscopy.1, 33 (1975)

[12] O. L. Krivanek, et.al. Nature.464.(2010).571-574.

[13] M.S.Isaacson, in Principles and Techniques of Electron Microscopy VIII, ed. M. Hayat (Van Nostrand-Reinhold, New York, 1976), pp. 1-78.

[14] K. Suenaga, et.al. Nature Chem. 1, 415 (2009).

[15] O.L.Krivanek, et.al., Ultramicrosopy.110, 935 (2010)

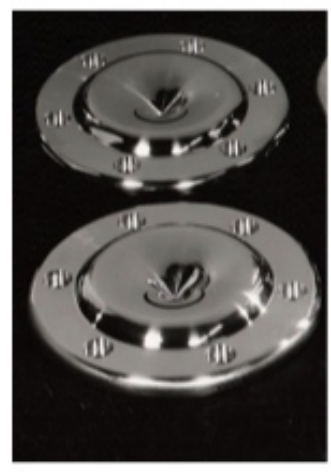

Fig.1: Butler gun anodes

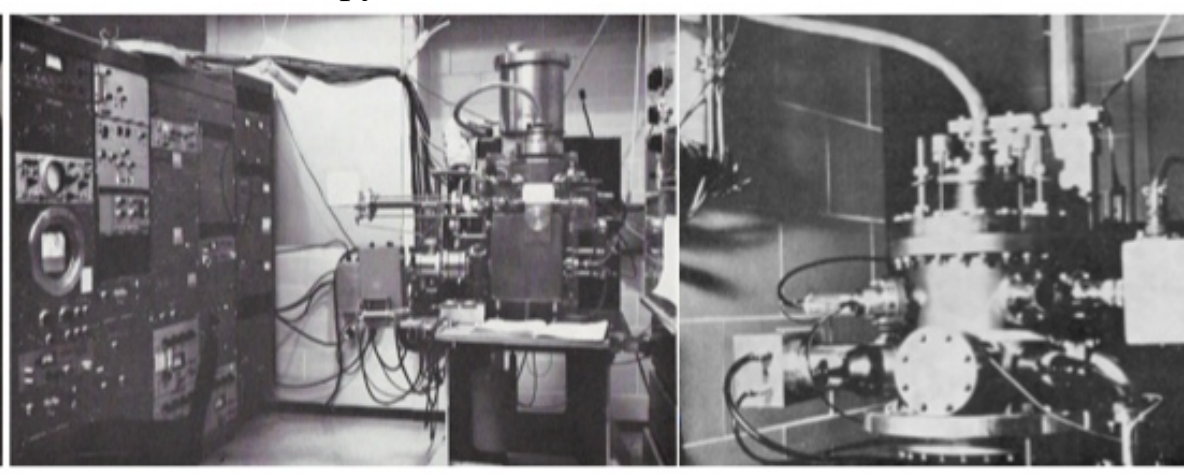

Fig.2: Univ. of Chicago 5 Angstrom STEM, circa 1966
Fig.3: Univ. of Chicago, "Gun" microscope

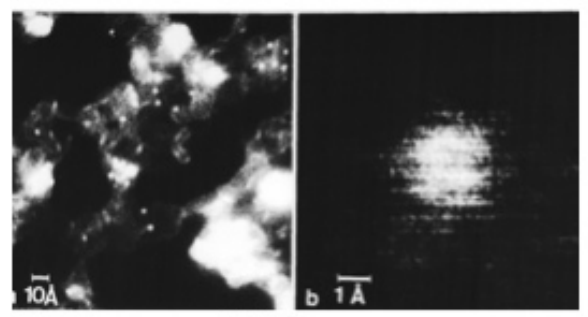

Fig.4: (left) Individual Au atoms on a $1 \mathrm{~nm}$ thick carbon substrate. b. scan over individual $\mathrm{Au}$ atom showing electrical and mechanica instabilities. ADF signal at $40 \mathrm{keV}$.

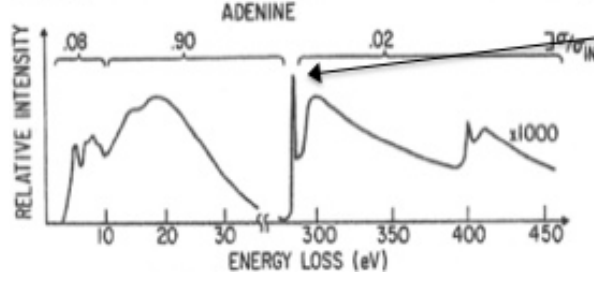

Fig.6 (left): Full EELS spectra of adenine taken with "Gun" microscope at

Fig.5(right): Inner shell EELS spectra "Gun" microscope at $25 \mathrm{keV}$. $25 \mathrm{keV}$.

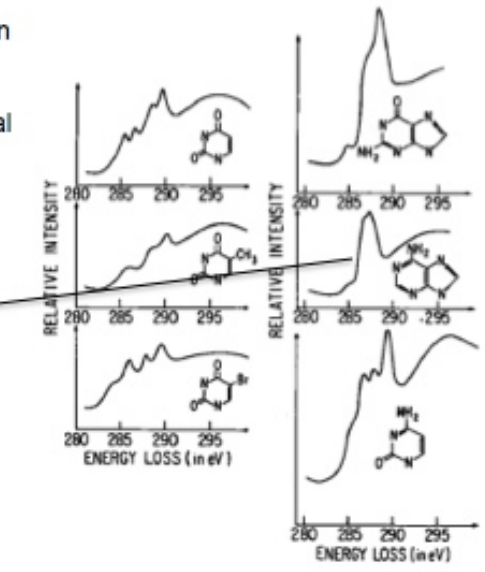

